\title{
OPEN Development and validation of LAMP primer sets for rapid identification of Aspergillus fumigatus carrying the cyp51A $\mathrm{TR}_{46}$ azole resistance gene
}

\author{
Plinio Trabasso ${ }^{1,6 \bowtie}$, Tetsuhiro Matsuzawa ${ }^{2}$, Teppei Arai ${ }^{3}$, Daisuke Hagiwara ${ }^{3,4,5}$, \\ Yuzuru Mikami ${ }^{3}$, Maria Luiza Moretti ${ }^{1} \&$ Akira Watanabe $^{3}$
}

Infections due to triazole-resistant Aspergillus fumigatus are increasingly reported worldwide and are associated with treatment failure and mortality. The principal class of azole-resistant isolates is characterized by tandem repeats of 34 bp or 46 bp within the promoter region of the cyp51A gene. Loop-mediated isothermal amplification (LAMP) is a widely used nucleic acid amplification system that is fast and specific. Here we describe a LAMP assay method to detect the $46 \mathrm{bp}$ tandem repeat insertion in the cyp51A gene promoter region based on novel LAMP primer sets. It also differentiated strains with $T_{46}$ tandem repeats from those with $T_{34}$ tandem repeats. These results showed this $T R_{46}{ }^{-}$ LAMP method is specific, rapid, and provides crucial insights to develop novel antifungal therapeutic strategies against severe fungal infections due to $A$. fumigatus with $\mathrm{TR}_{46}$ tandem repeats.

Antimicrobial resistance (AMR) was defined by the World Health Organization (WHO) as one of the most critical threats to human health. AMR can compromise our ability to treat infectious diseases, as well as undermining other advances in health care ${ }^{1}$. Although bacterial resistance remains the most common finding in the clinical setting, fungal resistance, especially to azole drugs among filamentous fungi, is relentlessly increasing worldwide ${ }^{2-4}$. Resistance to azole antifungals can be due, in general, to two major genetic mechanisms; point mutation(s) in the cyp51A open reading frame with or without tandem repeat (TR) of 34 or 46 base pair (bp) in the promoter region of the gene $\left(\mathrm{TR}_{34}\right.$ or $\left.\mathrm{TR}_{46}\right)$ and overexpression of oligonucleotide sequence in the cyp51A gene ${ }^{5}$. These mutations and overexpression of the gene confer different levels of resistance ${ }^{6}$. Point mutations result from previous exposure to azole drugs in a clinical setting, such as prophylaxis or therapeutic purposes ${ }^{7,8}$. On the other hand, TR with a point mutation(s) is the aftermath of previous exposure to azole fungicides. In an agricultural setting, azole fungicides are widely used to prevent fungal contamination in a large variety of crop and plant protection, allowing the TR-type resistant strains to emerge in the environments, and the conidia disperse to the air $^{8,9}$. In Brazil, agribusiness represents about $25 \%$ of the Brazilian Gross National Product (GNP) (http://www. agricultura.gov.br), and in this scenario, fungicide use has steadily increased over the years. The consumption of pesticides in Brazil grew 190\% in 2010, and fungicides corresponded to 14\% of this (https://www.ipessp.edu.br).

In the Netherlands, the prevalence of TR-type resistant strains was high. Of 952 clinical A. fumigatus strains were collected and included 225 and 98 had $\mathrm{TR}_{34}$ and $\mathrm{TR}_{46}$, respectively ${ }^{10}$. In another study, $\mathrm{TR}_{34} \mathrm{~L}_{98 \mathrm{H}}$ and $\mathrm{TR}_{46}$ Y121F T289A mutation that occur in patients without previous azole exposure have been reported in Europe, Asia, the Middle East, Africa, and Australia ${ }^{11}$.

Additionally, there are several reports of fatal invasive aspergillosis caused by A. fumigatus carrying the $\mathrm{TR}_{46}$ in acute myeloid leukemia (AML) patients and hematopoietic stem cell transplant recipients ${ }^{12}$. Higher mortality

\footnotetext{
${ }^{1}$ School of Medical Sciences, University of Campinas, Campinas, Sao Paulo, Brazil. 'University of Nagasaki, Nagasaki, Japan. ${ }^{3}$ Medical Mycology Research Center, Chiba University, Chiba, Japan. ${ }^{4}$ Faculty of Life and Environmental Sciences, University of Tsukuba, Tsukuba, Ibaraki, Japan. ${ }^{5}$ Microbiology Research Center for Sustainability, University of Tsukuba, Ibaraki, Japan. ${ }^{6}$ Department of Internal Medicine, School of Medical Sciences, University of Campinas, Rua Tessalia Vieira de Camargo, Campinas, Sao Paulo 126, Brazil. ${ }^{\square}$ email: trabasso@ unicamp.br
} 
of patients with invasive aspergillosis caused by azole-resistant strains has been reported ${ }^{12,13}$. Thus, a rapid and specific method to identify the presence of TR would contribute to faster therapeutic decision-making ${ }^{14}$.

As one of the promising diagnostic tools for the azole-resistant A. fumigatus, loop-mediated isothermal amplification (LAMP) for the development of improved DNA-based diagnostic kits has been reported ${ }^{15}$. In general, the LAMP method was found to be similar or superior to the standard PCR method, more specific, lower-cost, and easier to perform. LAMP-based approaches have been applied to a wide range of samples, such as whole blood, paraffin-embedded tissues, and various microbial pathogens ${ }^{16,17}$. In this paper, we report a novel LAMP assay method that selectively detects triazole resistant $A$. fumigatus strains due to the presence of double $\mathrm{TR}_{46}\left(\mathrm{TR}_{46}{ }^{2}\right)$ or triple $\mathrm{TR}_{46}\left(\mathrm{TR}_{46}{ }^{3}\right)$ in the cyp51A promoter region.

\section{Results}

Antifungal susceptibility tests. Drug susceptibilities of 41 A. fumigatus strains against azole drugs itraconazole and voriconazole are shown in Table 1. Thirty strains designated as wild type were isolated from clinical specimens, and they were confirmed to be susceptible to itraconazole and voriconazole. The remaining 11 strains $\left(\mathrm{TR}_{34}\right.$ and $\left.\mathrm{TR}_{46}\right)$ were resistant to voriconazole, and most of them showed MIC values of $>8 \mu \mathrm{g} / \mathrm{mL}$ against voriconazole. Among the 11 strains, 2 strains (IFM64460 with TR34/L978H and IFM64733 with TR34/LH98H) were resistant to itraconazole, and the remaining 9 strains were susceptible to itraconazole (Table 1).

Primer design. The most crucial step in the LAMP assay is the design of primers. In the LAMP assay, six primers are necessary to amplify the targeted region under isothermal condition. First, we inspected the promoter region ( $-461 \mathrm{bp}$ to $-296 \mathrm{bp}$ counted from start codon) of the cyp51A gene to select a set of primer sequences that specifically amplify the repeated 46 bp sequence in strains with a $\mathrm{TR}_{46}$ mutation (Figs. 1 and 2 ). To enable specific amplification against repeated $\mathrm{TR}_{46}$ sequences, $\mathrm{B} 2$ was set on the joint of two 46 bp sequences. Then, another five sequences for primer sets were chosen in the target region, according to the standard criteria, to obtain a specific and rapid LAMP primer set in the LAMP assay. Namely, six primers (F1, F2, F3, B1, B2, B3) that target six specific regions of a DNA template of the $\mathrm{TR}_{46}$ gene of $c y p 51 A$ were selected, and in addition, two loop primers (LF, LB) were also chosen to accelerate the reaction (Fig. 1). Several new candidates of LAMP primers were designed based on the above information and their utility tested. From those, one useful LAMP primer set based on the detection of $\mathrm{TR}_{46}$ regions in the cyp51A gene was selected (Table 2). In this LAMP method, the primers were selected based on the criteria that amplification started within about 30-50 min, and maximum amplification was completed within 70-80 min. Nucleotide sequence of promoter region for resistance gene of LAMP primer sets to detect the resistance gene was shown in Fig. 3. This primer amplifies between consecutive $46 \mathrm{bp}$ sequences between $\mathrm{TR}_{46}-1 \mathrm{bp}$ and $\mathrm{TR}_{46}-2 \mathrm{bp}$. The base sequence in this part corresponds to the $\mathrm{B} 2$ sequence in Table 2.

Validation of LAMP primer sets for $\mathbf{T R}_{\mathbf{4 6}}$. The specificity of the primer sets was tested using various types of A. fumigatus strains, such as wild isolates and environmental or clinical azole resistance isolates (Fig. 4). In this study, IFM63432 and IFM62918 strains were used as positive and negative control strains, respectively. As shown in Fig. 4A-i, TR46 LAMP primer could not amplify the DNA from 30 strains of azole drug-susceptible clinical isolates of A. fumigatus. However, the start of the LAMP amplification in the positive control strain of A. fumigatus strains (IFM 63432) was at around $50 \mathrm{~min}$. On the other hand, $\mathrm{TR}_{46}$ LAMP primer could amplify DNA from A. fumigatus strains carrying the duplicate 46 bp promoter repeat in cyp51A gene (IFM63432, BE1-2, BE1-4, BE3-5, BE3-6) as shown in Fig. 4A-ii. This result suggests that the present LAMP primer could amplify the four $\mathrm{TR}_{46}{ }^{2}$ strains harboring the TR46 resistant mutation (TR46/Y121F/T289A). It was also confirmed that $\mathrm{TR}_{46}$ LAMP primer could amplify DNA of A. fumigatus strains carrying three tandem repeats $\left(\mathrm{TR}_{46}{ }^{3}\right)(\mathrm{BE} 1-1$, W1-4, W2-12-1) (Fig. 4A-iii). When this $\mathrm{TR}_{46}$ LAMP primer was tested for three $\mathrm{TR}_{34}{ }^{2}$ strains (Table 1), namely strains IFM64460 and IFM64733 (with mutation of $\mathrm{TR}_{34} / \mathrm{L} 98 \mathrm{H}$ ) and strain 3-1-B (with mutations of TR Th $_{34} / \mathrm{L}^{2} \mathrm{HH} /$ Y289/T289A/I364V/G448S), DNA amplification was not observed (Fig. 4B-i,B-ii). These results also suggested that the present LAMP primer could not detect $\mathrm{TR}_{34}{ }^{2}$ drug-resistant strains regardless of their point mutation site in the cyp51A gene (Fig. 4B-i,B-ii). These studies confirmed that the newly established $\mathrm{TR}_{46}$ LAMP primer set was specific for A. fumigatus strains with TR of double or triple 46-bp promoter tandem repeats in the cyp51A gene. The sensitivity of the $\mathrm{TR}_{46}$ LAMP assay was verified. The detection limit was $1 \times 10^{4}$ copies per reaction in $60 \mathrm{~min}$. In the $80 \mathrm{~min}$ reaction, $10^{2}$ copies per reaction were also detected (Fig. 5).

\section{Discussion}

Azole antifungals mainly inhibit the ergosterol biosynthetic pathway by targeting the cytochrome P450-dependent enzyme lanosterol 14-a-demethylase, encoded by cyp51A in molds. Resistance to this class of drugs in the major human pathogen A. fumigatus is emerging and reaching levels to prevent their clinical use ${ }^{6}$. Advances in recent molecular genetic technologies such as real-time PCR have introduced various proper diagnostic assay methods into the fields in azole-resistant mechanism analysis. The LAMP assay described here has advantages of high sensitivity and specificity, low costs, and short amplification time. In addition, there have been no reports using LAMP techniques to study azole-resistant mechanisms in A. fumigatus by the strains with $\mathrm{TR}_{46}$ in the cyp51A promoter region.

Recently Yu Shan-Ling et al. ${ }^{18}$ reported a similar rapid technique to detect azole-resistant strains due to amplification of a TR of a $34 \mathrm{bp}\left(\mathrm{TR}_{34}\right)$ and a $46 \mathrm{bp}\left(\mathrm{TR}_{46}\right)$ within the promoter region of cyp51A of A. fumigatus. However, here we used a newly designed $\mathrm{TR}_{46}$ LAMP primer set different from those reported by Yu Shan-Ling et al. ${ }^{18}$. Compared to experiments such as Yu Shan-Ling et al. ${ }^{18}$, our method used adjusted genomic DNA (2 ng/ 


\begin{tabular}{|c|c|c|c|c|}
\hline \multirow[b]{2}{*}{ Strain no } & \multirow[b]{2}{*}{ Isolation sources } & \multirow[b]{2}{*}{ cyp51A genotypes } & \multicolumn{2}{|c|}{$\begin{array}{l}\text { MIC values }(\mu \mathrm{g} \\
\mathrm{ml})\end{array}$} \\
\hline & & & ITCZ & VRCZ \\
\hline IFM63432 ${ }^{\mathrm{a}}$ & Clinic & $\mathrm{TR}_{46}{ }^{2} / \mathrm{Y} 121 \mathrm{~F} / \mathrm{T} 289 \mathrm{~A}$ & 4 & $>8$ \\
\hline BE1-2 & Environment (bulb) ${ }^{\mathrm{b}}$ & $\mathrm{TR}_{46}{ }^{2} / \mathrm{Y} 121 \mathrm{~F} / \mathrm{T} 289 \mathrm{~A}$ & 2 & $>8$ \\
\hline BE1-4 & Environment (bulb) & $\mathrm{TR}_{46}{ }^{2} / \mathrm{Y} 121 \mathrm{~F} / \mathrm{S} 363 \mathrm{P} / \mathrm{I} 364 \mathrm{~V} / \mathrm{G} 448 \mathrm{~S}$ & 2 & $>8$ \\
\hline BE 3-5 & environment (bulb & $\mathrm{TR}_{46}^{2} / \mathrm{Y} 121 \mathrm{~F} / \mathrm{T} 289 \mathrm{~A}$ & 2 & $>8$ \\
\hline BE 3-6 & Environment (bulb) & $\mathrm{TR}_{46}{ }^{2} / \mathrm{Y} 121 \mathrm{~F} / \mathrm{T} 289 \mathrm{~A}$ & 2 & $>8$ \\
\hline BE 1-1 & Environment (bulb) & $\mathrm{TR}_{46}{ }^{3} / \mathrm{Y} 121 \mathrm{~F} / \mathrm{M} 172 \mathrm{I} / \mathrm{T} 289 \mathrm{~A} / \mathrm{G} 448 \mathrm{~S}$ & 2 & $>8$ \\
\hline W1-4 & Environment (bulb) & $\mathrm{TR}_{46}{ }^{3} / \mathrm{Y} 121 \mathrm{~F} / \mathrm{M} 172 \mathrm{I} / \mathrm{T} 289 \mathrm{~A} / \mathrm{G} 448 \mathrm{~S}$ & 2 & $>8$ \\
\hline W2-12-1 & Environment (bulb) & $\mathrm{TR}_{46}{ }^{3} / \mathrm{Y} 121 \mathrm{~F} / \mathrm{M} 172 \mathrm{I} / \mathrm{T} 289 \mathrm{~A} / \mathrm{G} 448 \mathrm{~S}$ & 2 & $>8$ \\
\hline IFM64460 & Clinic & $\mathrm{TR}_{34} / \mathrm{L} 98 \mathrm{H}$ & $>8$ & $>8$ \\
\hline IFM64733 & Environment & $\mathrm{TR}_{34} / \mathrm{L} 98 \mathrm{H}$ & $>8$ & $>8$ \\
\hline $3-1-B$ & Environment (bulb) & $\mathrm{TR}_{34} / \mathrm{L} 98 \mathrm{H} / \mathrm{T} 289 \mathrm{~A} / \mathrm{I} 364 \mathrm{~V} / \mathrm{G} 448 \mathrm{~S}$ & 2 & $>8$ \\
\hline IFM62918c & Clinic & Wild & 0.5 & 1 \\
\hline IFM62799 & Clinic & Wild & 0.5 & 1 \\
\hline IFM60516 & Clinic & Wild & 1 & 1 \\
\hline IFM58402 & Clinic & Wild & 0.5 & 0.5 \\
\hline IFM51977 & Clinic & Wild & 0.25 & 0.25 \\
\hline IFM60065 & Clinic & Wild & 1 & 0.5 \\
\hline IFM61960 & Clinic & Wild & 0.5 & 0.5 \\
\hline IFM51748 & Clinic & Wild & 0.125 & 0.125 \\
\hline IFM63666 & Clinic & Wild & 1 & 2 \\
\hline IFM62520 & Clinic & Wild & 1 & 0.5 \\
\hline IFM50999 & Clinic & Wild & 0.5 & 0.5 \\
\hline IFM50268 & Clinic & Wild & 0.25 & 0.125 \\
\hline IFM55548 & Clinic & Wild & 0.25 & 0.25 \\
\hline IFM63311 & Clinic & Wild & 1 & 0.5 \\
\hline IFM63355 & Clinic & Wild & 2 & 2 \\
\hline IFM60901 & Clinic & Wild & 0.5 & 0.5 \\
\hline IFM62674 & Clinic & Wild & 1 & 2 \\
\hline IFM62709 & Clinic & Wild & 0.5 & 0.5 \\
\hline IFM52659 & Clinic & Wild & 1 & 1 \\
\hline IFM57130 & Clinic & Wild & 0.25 & 0.125 \\
\hline IFM60814 & Clinic & Wild & 0.5 & 0.5 \\
\hline IFM49435 & Clinic & Wild & 0.25 & 0.25 \\
\hline IFM61572 & Clinic & Wild & 0.5 & 0.5 \\
\hline IFM50669 & Clinic & Wild & 0.5 & 0.25 \\
\hline IFM59832 & Clinic & Wild & 0.5 & 0.5 \\
\hline IFM55044 & Clinic & Wild & 0.25 & 0.25 \\
\hline IFM47670 & Clinic & Wild & 0.5 & 0.5 \\
\hline IFM51978 & Clinic & Wild & 0.5 & 0.25 \\
\hline IFM58328 & Clinic & Wild & 0.5 & 0.5 \\
\hline IFM60369 & Clinic & Wild & 0.5 & 0.5 \\
\hline
\end{tabular}

Table 1. Aspergillus fumigatus strains used in this experiment and their drug susceptibility profiles against itraconazole and voriconazole. ITCZ itraconazole, $V R C Z$ voriconazole. ${ }^{a} L A M P$ positive control strain. ${ }^{\mathrm{b}}$ obtained from plant bulbs. ${ }^{\mathrm{c}}$ LAMP negative control strain.

$\mu \mathrm{L}$ ) and can detect $\mathrm{TR}_{46}$ strains when resistance is confirmed, and this leads to a simple identification method of A. fumigatus carrying the $\mathrm{TR}_{46}$ in the cyp51A promoter region, in routine clinical practice.

There is a difference in MIC values between strains with $\mathrm{TR}_{46}$ and strains with $\mathrm{TR}_{34}{ }^{19}$. Therefore, the importance of detecting $\mathrm{TR}_{46}$ lies in the fact that strains of $A$. fumigatus harboring $\mathrm{TR}_{46}$ are resistant to voriconazole but not to itraconazole. Two $\mathrm{TR}_{34}$ strains (IFM64460: $\mathrm{TR}_{34} / \mathrm{L} 98 \mathrm{H}$ and IFM64733: $\mathrm{TR}_{34} / \mathrm{L} 98 \mathrm{H}$ ) are highly resistant to voriconazole ${ }^{19}$ but not to itraconazole. Further detailed drug susceptibility mechanism study against $\mathrm{TR}_{34}$ strain (3-1-B: $\left.\mathrm{TR}_{34} / \mathrm{L} 98 \mathrm{H} / \mathrm{T} 289 \mathrm{~A} / \mathrm{I} 364 \mathrm{~V} / \mathrm{G} 448 \mathrm{~S}\right)$ is of interest.

The high specificity and rapidity of the LAMP assay are achieved by applying four primers that target six regions of a DNA template, and two loop primers (LF, LB) to accelerate the reaction. In this study, we succeeded in designing valuable $\mathrm{TR}_{46}$ LAMP primer sets to detect specifically a $\mathrm{TR}_{46}$ within the promoter regions 


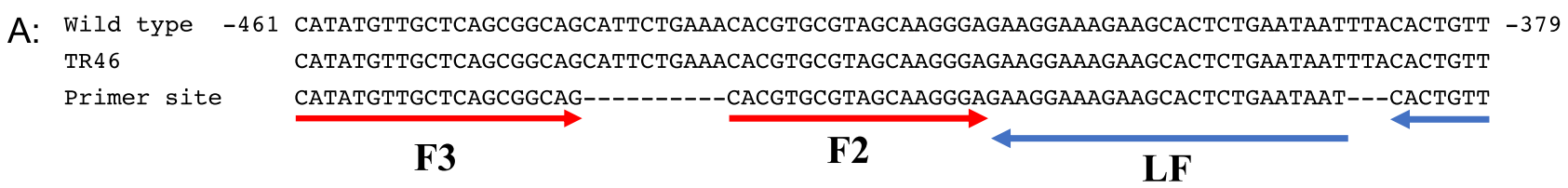

Wild type -378 CTCCTCTAGAAAAAACTCATGAGTGAATAATCGCAGCACCACTTCAGAGTTGTCTAGAATCACGCGGTCCGGATGTGTGCTGA -296 TR4 6 CTCCTCTAGAAAAAACTCATGAGTGAATAATCGCAGCACCACTTCAGAGTTGtctagaatcacgcggtccggatgtgtgctga

Primer site СТССТСTAGAAAAAACTC-

\section{F1}

B1C

LB

Wild type -295

TR4 6

gccgaatgaaagttgTCTAGAATCACGCGGTCCGGATGTGTGCTGAGCCGAATGAAAGTTGCCTAATTACTAAG

Primer site

B3C

B:

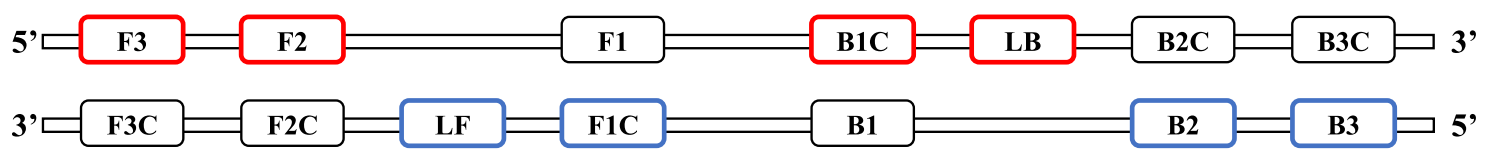

Figure 1. Genetic information for the design of the LAMP primer sets. (A) Schematic illustration of cyp51A gene showing LAMP primer positions and corresponding sequences of TR46 bp promoter tandem repeat compared to wild-type sequences. (B) Primers F3, F2, F1, B1, B2, and B3 show primer sequence positions. Sequences of some primers are complementary, as shown in Table 2. See LAMP primer and methods, which are shown in Refs. ${ }^{19,20}$.

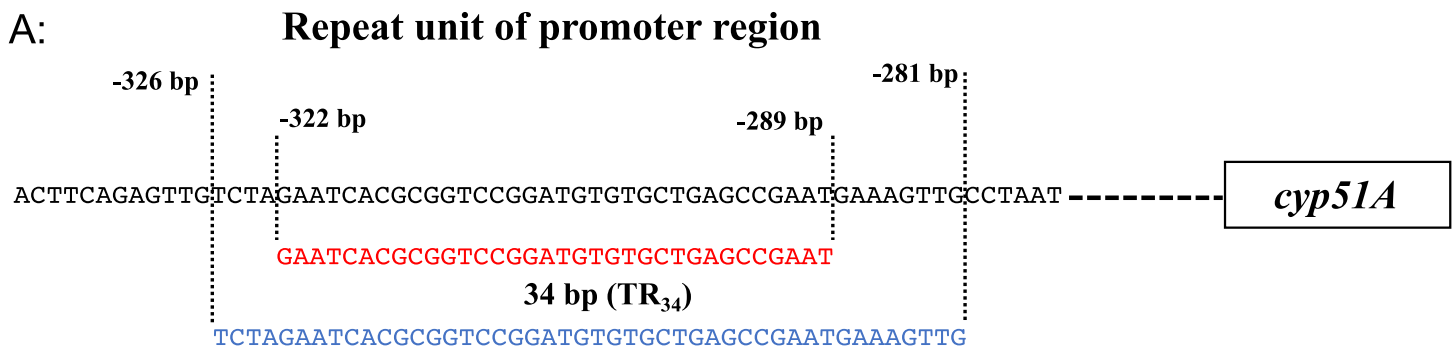

46 bp $\left(\mathrm{TR}_{46}\right)$

B: cyp51A promoter region azole resisitance mutations in the coding region

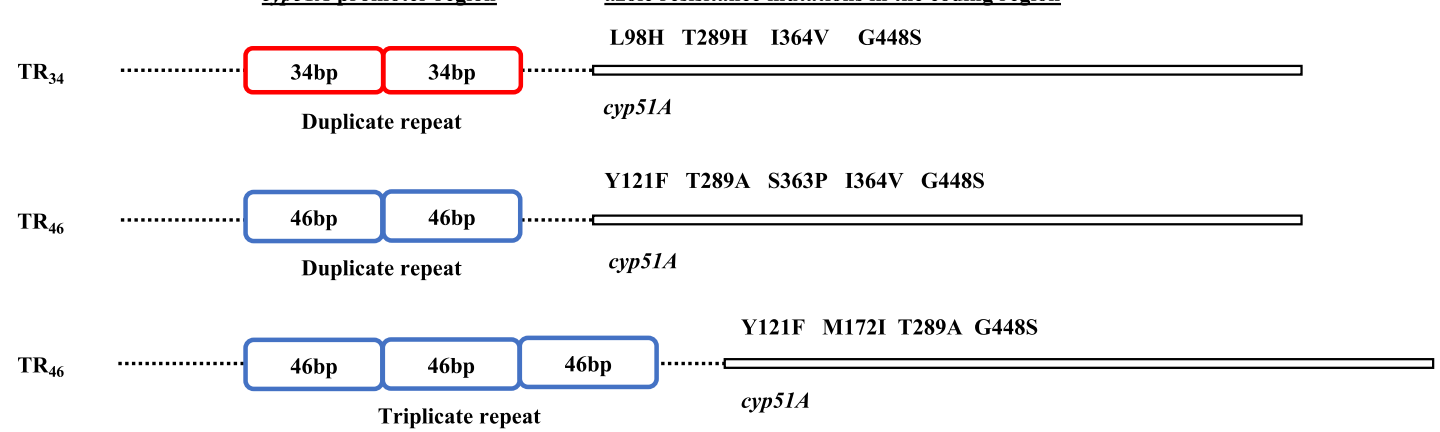

Figure 2. Illustration of tandem repeat regions of cyp51A genes used in this experiment. (A) Tandem repeat unit of promoter genes of $\mathrm{TR}_{34}$ and $\mathrm{TR}_{46}$. (B) Tandem repeat: $34 \mathrm{bp}$ (double) and $46 \mathrm{bp}$ (double or triple), and cyp51A gene associated point mutation place. 


\begin{tabular}{|l|l|}
\hline LAMP primer names & Sequence $\left(\mathbf{5}^{\prime}\right.$ to $\left.\mathbf{3}^{\prime}\right)$ \\
\hline F3 & CATATGTTGCTCAGCGGCAG \\
\hline B3 & CAACTTTCATTCGGCTCAGCA \\
\hline FIP (F1 complementary+F2) & GAGTTTTTTCTAGAGGAGAACAGTG-CACGTGCGTAGCAAGGGA \\
\hline BIP (B1 + B2 complementary) & CACCACTTCAGAGTTGTCTAGAAT-ACCGCGTGATTCTAGACAACTTTC \\
\hline LF & ATTATTCAGAGTGCTTCTTTCCTTC \\
\hline LB & GGTCCGGATGTGTGCTG \\
\hline
\end{tabular}

Table 2. Sequence information of newly designed TR46-LAMP primer sets in the present experiment.

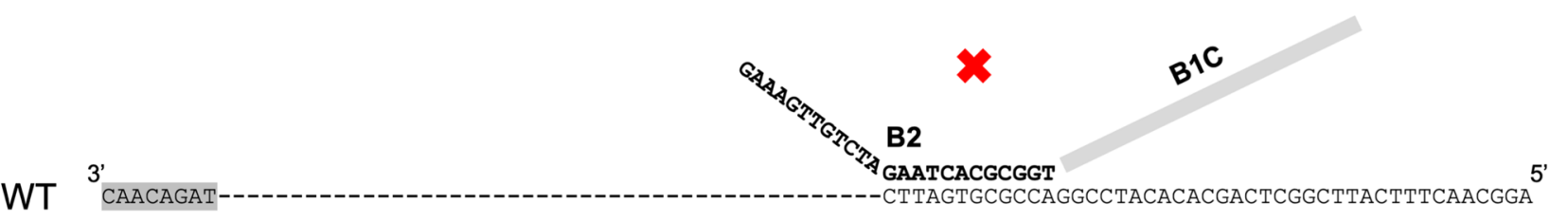

TR3 $34^{3}$

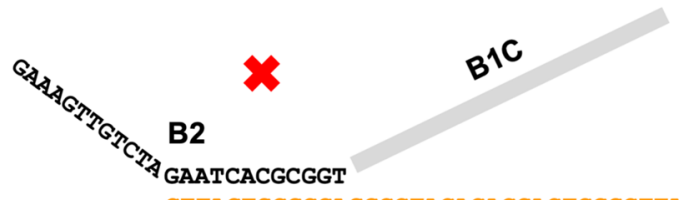

CAACAGATCTTAGTGCGCCAGGCCTACACACGACTCGGCTTA-

TR34-1

TR34-2

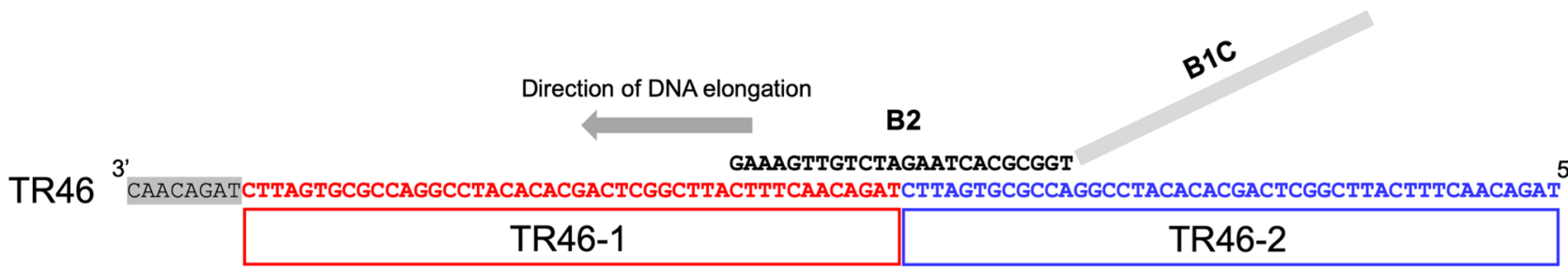

Figure 3. Schematic figure of $\mathrm{TR}_{46}$ LAMP primer amplification site in comparison with those of wild type and $\mathrm{TR}_{34}$. The nucleotide sequence is targeted for the promoter region for the $\mathrm{TR}_{46}$ resistance gene (between $\mathrm{TR}_{46}-1$ and $\mathrm{TR}_{46}-2$ ). The sequence in this part corresponds to the $\mathrm{B} 2$ sequence in Table 2.

of azole-resistant $A$. fumigatus. Furthermore, the designed primer sets could differentiate azole-resistant $\mathrm{TR}_{46}$ strains from the $\mathrm{TR}_{34}$ strains and wild type strains. There was no cross reaction of the assay with neither the $\mathrm{TR}_{34}$ type nor the wild type. Further studies on the preparation of specific primers that can distinguish between $\mathrm{TR}_{34}$ strains and wild type strains regardless of the results of drug susceptibility testing are needed. To our knowledge, this is a new and helpful report of a detection method for one of the most prevalent cyp51A resistant gene $\mathrm{TR}_{46}$ in A. fumigatus azole-resistant strains.

Recently, the strain consisting of the four repeats of $46 \mathrm{bp}$ of the promoter region was reported in the Netherlands $\left(\mathrm{TR}_{46}{ }^{4}\right)^{20}$. The LAMP primer we designed was able to detect both two copies of the TR46 tandem repeat and three copies of the $\mathrm{TR}_{46}$. Moreover, these amplification curves (as well as the starting point) were similar. The $\mathrm{BIP}(\mathrm{B} 1+\mathrm{B} 2$ complementary; Fig. 1) of the primer we designed is TR-specific. B1 is designed at the boundary where the repeat unit is inserted, and B2 is designed at the boundary between the repeat units. In addition, B3 is designed on a repeat unit. Based on the results of strains having double repeat and triple repeat, it was suggested that the primer used this time may be able to detect even if the number of repeats increases, such as $\mathrm{TR}_{46}{ }^{4}$.

It is widely known that exposure to azole fungicides resulted in the emergence of azole-resistant strains with tandem repeats in the promoter region of $c y p 51 A$ gene $^{8,9}$. For this reason, epidemiological studies such as the incidence of azole-resistant strains in the environment are essential. Many environmental and clinical isolates need to be screened to generate epidemiological data, such as the frequency of detection of azole-resistant $A$. fumigatus. The method developed in this study would be an easy-to-use screening procedure.

Since the LAMP assay developed in the present study is a one-step and rapid detection method, coupled with its high reliability and ease of use, it can prompt detect specific drug-resistant genes due to $\mathrm{TR}_{46}$ in $A$. fumigatus in the clinical laboratory setting. Thus, early detection of infections due to $\mathrm{TR}_{46}$ drug-resistant strains in $A$. fumigatus might be helpful to guide the early start of corrective and effective antifungal therapy. 
A

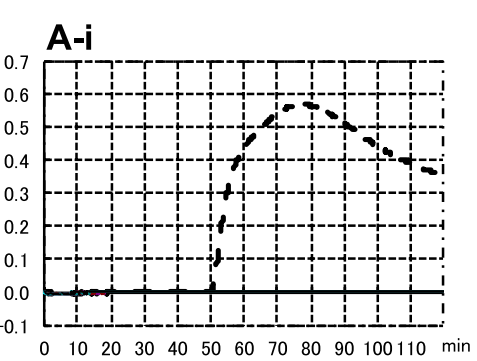

B

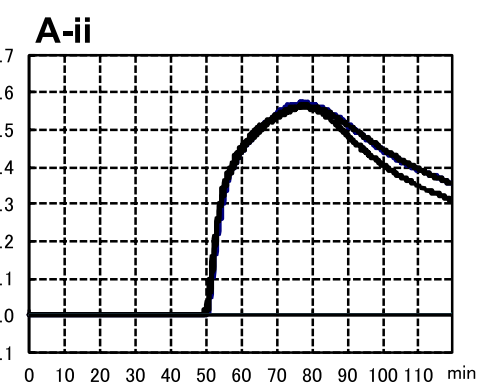

B-i

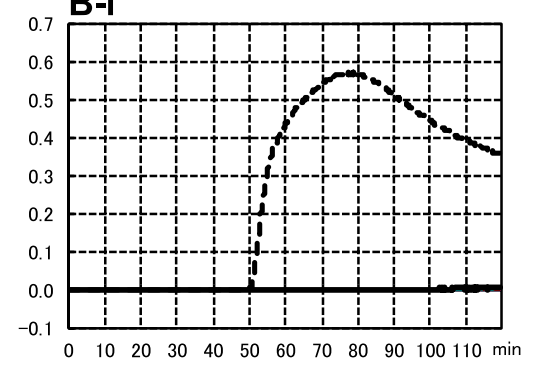

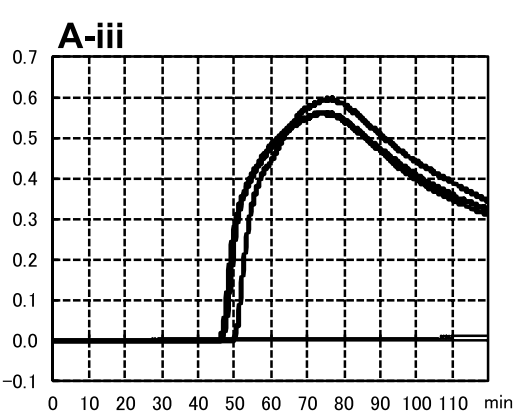

B-ii

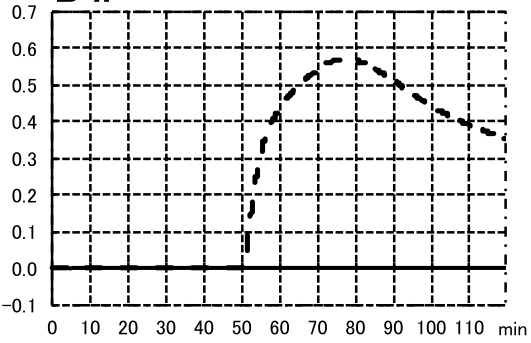

Figure 4. Comparative amplification profiles of A. fumigatus wild type and environmental or clinical azoleresistant isolates with or without TR46 double or triple 46 bp promoter repeats in cyp51A gene by a newly developed LAMP primer sets. The dotted curve shows the amplification by control strain (IFM 63432). (A-i) DNA amplification profiles using 30 strains of A. fumigatus wild type. DNA amplification was not confirmed in all wild-type strains tested (30 strains). Among 30 wild-type strains, IFM 62918 strain was used as a negative control strain (no amplification). (A-ii) DNA amplification was confirmed by five $\mathrm{TR}_{46}{ }^{2}$ strains (IFM63432, BE1-2, BE1-4, BE3-5, BE3-6), which have double 46 bp promoter repeats. (A-iii) DNA amplification was confirmed by three $\mathrm{TR}_{46}{ }^{3}$ strains (BE1-1, W1-4, W2-12-1), which have triple 46 bp promoter repeats. (B-i) DNA amplification was not confirmed by two $\mathrm{TR}_{34}{ }^{2}$ strains (IFM64460, IFM64733), which have duplicate 34 bp promoter repeats with one mutation in the one coding region $(\mathrm{L} 98 \mathrm{H})$. The dotted line shows amplification by the control strain. (B-ii) DNA amplification was not confirmed by one $\mathrm{TR}_{34}{ }^{2}$ strain (3-1-B), which has duplicate $34 \mathrm{bp}$ promoter repeats with multi-mutations in the four coding regions (L98H/T289A/I364V/G448S). The dotted line shows amplification by the control strain.

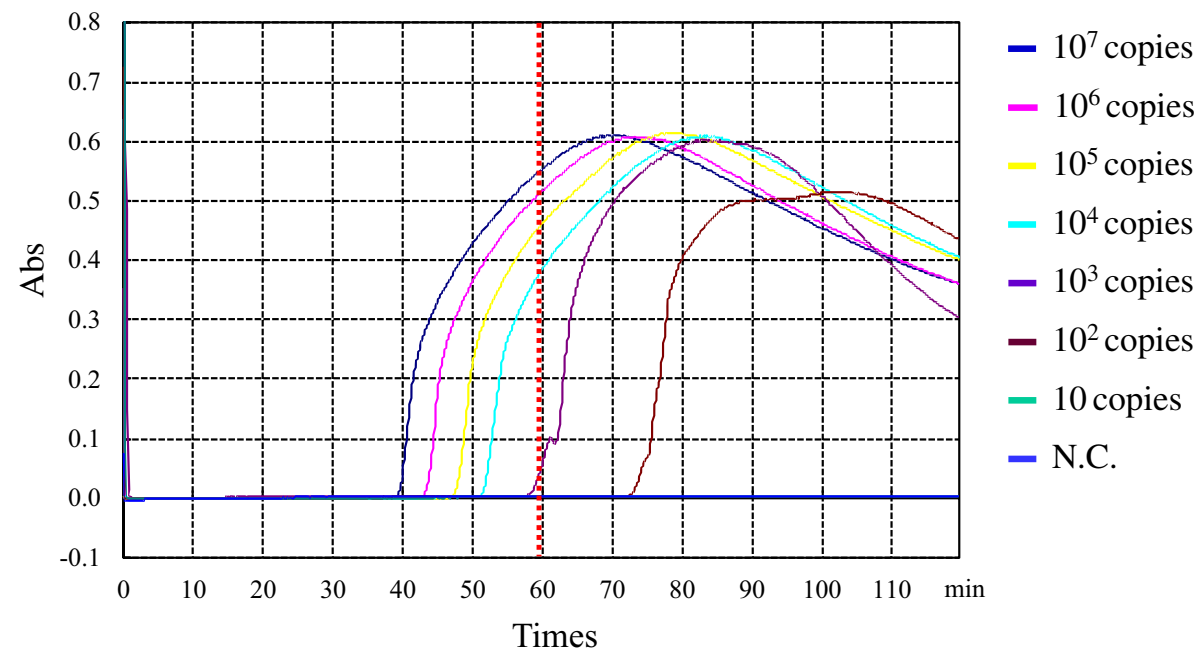

Figure 5. Experimental results of the detection limit of the $\mathrm{TR}_{46} \mathrm{LAMP}$ assay. The detection limit reaction was carried out using $10^{7}$ to 10 copies of plasmid DNA per reaction. The detection limit was measured within $60 \mathrm{~min}$. 


\section{Methods}

Aspergillus isolates and MIC determination by broth microdilution test. Forty-one strains, including thirty-three from the clinical setting and eight environmental (plant bulbs) isolates ${ }^{21}$ of A. fumigatus, were provided through the National Bio-Resource Project (NBRP), Japan (http://www.nbrp.jp/); source and drug susceptibility are shown in Table 1.

DNA preparation and extraction. Fungal strains were cultured on Sabouraud dextrose agar. Genomic DNA was extracted from overnight cultures of A. fumigatus mycelia by the urea-phenol method. Mycelia were mixed with $0.5 \mathrm{~mm}$ size glass beads, $0.5 \mathrm{ml}$ of PCI (phenol/chloroform/Isoamyl alcohol) solution and $0.5 \mathrm{ml}$ DNA extraction buffer (50 mM Tris- $\mathrm{HCl}, \mathrm{pH} 8.0,20 \mathrm{mM}$ EDTA, $0.3 \mathrm{M} \mathrm{NaCl}, 0.5 \%$ SDS, $5 \mathrm{M}$ urea), and disrupted by Fast Prep FP100A (MP-Biomedicals, Santa Ana, USA) for 3 cycles of $30 \mathrm{~s}$ each at a speed of $4.0 \mathrm{~m} / \mathrm{s}$. After centrifugation, the upper layer was transferred to a new tube and subjected to ethanol precipitation. The resulting DNA pellet was suspended in $100 \mu \mathrm{L}$ TE buffer. DNA concentration was determined by the methods described in our previous paper ${ }^{22}$.

All A. fumigatus strains were submitted to antifungal susceptibility tests according to the CLSI M38 protocol (https://clsi.org/standards/products/microbiology/documents/m38/), using Eiken Dried Plates (9DEF47, Eiken Chemical Co., Tokyo, Japan).

LAMP-method. LAMP was performed as described in our previous studies ${ }^{23}$. $\mathrm{TR}_{46}$ LAMP primers were designed based on the target promoter region sequences of the cyp51A gene of A. fumigatus, which includes tandem repeats in the promoter region containing $\mathrm{TR}_{46}$ mutant alleles. The sequence of the $c y p 51 \mathrm{~A}$ gene was downloaded from NCBI Gen-Bank (https://www.ncbi.nlm.nih.gov/genebank, accession numbers AF222068 for wild type, $\mathrm{MH} 231595.1$ for $\mathrm{TR}_{34}$, and MH040305.1 for $\mathrm{TR}_{46}$ ). In total, a 184-bp nucleotide alignment (Fig. 1) was used for $\mathrm{TR}_{46}$ LAMP primer design by the protocol of the Eiken Company (Primer Explorer V5, Eiken Chemical Co. Ltd, Tokyo. Japan). LAMP primers are composed of six primers recognizing eight distinct regions. LAMP reactions were performed with a Loopamp DNA amplification kit using reaction mixtures composed of $40 \mathrm{pmol}$ each of primers FIP and BIP, 5 pmol each of primers F3 and B3, 20 pmol each of primers $\mathrm{LF}$ and LB, $12.5 \mathrm{~mL} \times 2$ reaction mixture, $1 \mu \mathrm{l}$ Bst DNA polymerase, $2 \mu \mathrm{L}$ DNA sample and distilled water up to a final volume of $25 \mu \mathrm{L}$ (Eiken Chemical Co., Ltd., Tokyo, Japan). The LAMP reactions were analyzed by a real-time turbidimeter (Loopamp EXIA; Eiken Chemical Co.) and were conducted at $63^{\circ} \mathrm{C}$, for $120 \mathrm{~min}$ and then heated at $80^{\circ} \mathrm{C}$ for $2 \mathrm{~min}$ to terminate the reaction. The start of amplification of LAMP products at 30 to $50 \mathrm{~min}$ in the graph suggested the positive reaction due to the presence of corresponding $46 \mathrm{bp}$ tandem repeats of cyp51A gene. Since overall reaction can be obtained within $2 \mathrm{~h}$, prompt drug therapy can be deployed within a short time. To check the detection limit of $\mathrm{TR}_{46}$ specific LAMP primers, the plasmid DNA was used. To construct plasmid contained $\mathrm{TR}_{46}$ and cyp51A gene sequences, we cloned the alleles using the shuttle vector pCB1004. Genomic DNA of IFM63432 was used as template to clone the alleles. The cyp51 coding region including approximately $1 \mathrm{~kb}$ fragments upstream and downstream were amplified by PCR using the primers pCB1004_Hind_cyp51A-F (5'-aggaattcgatatcaTAG AATGAGTGAGCTGATTT-3') and pCB1004_Kpn_cyp51A-R (5'-gggcgaattgggtacCAGGTTTTCGCACGA GCTTCTCC-3'). Amplified DNA fragments were fused into pCB1004 digested with HindIII and KpnI, by InFusion H Cloning Kit (Takara Bio, Otsu, Japan). The size of plasmid DNA was 8164 bp.

Received: 12 May 2021; Accepted: 9 August 2021

Published online: 24 August 2021

\section{References}

1. Revie, N. M., Lyer, K. R., Robbins, N. \& Cowen, L. E. Antifungal drug resistance: evolution, mechanisms and impact. Curr. Opin. Microbiol. 45, 70-76 (2018).

2. Chowdhary, A., Sharma, C., Hagen, F. \& Meis, J. F. Exploring azole antifungal drug resistance in Aspergillus fumigatus with particular reference to resistance mechanisms. Future Microbiol. 9, 697-711 (2014).

3. Beer, K. D. et al. Multidrug-resistant Aspergillus fumigatus carrying mutations linked to environmental fungicide exposure: Three states, 2010-2017. MMWR Morb. Mortal Wkly. Rep. 67, 1064-1067 (2018).

4. Patterson, T. E. et al. Practice guidelines for the diagnosis and management of aspergillosis: 2016 update by the Infectious Diseases Society of America. Clin. Infect. Dis. 63, 438-442 (2016).

5. Hagiwara, D. et al. Epidemiological and genomic landscape of azole resistance mechanisms in Aspergillus fungi. Front. Microbiol. 7, $1382(2016)$.

6. Enserink, M. Infectious diseases: Farm fungicides linked to resistance in a human pathogen. Science 326(5957), 1173. https://doi. org/10.1126/science.326.5957.1173 (2009).

7. Denning, D. W. et al. Chronic pulmonary aspergillosis: rationale and clinical guidelines for diagnosis and management. Eur. Respir. J. 47, 45-68 (2016).

8. Gsaller, F. et al. Sterol biosynthesis and azole tolerance is governed by the opposing actions of SrbA and the CCAT binding complex. PLOS Pathog. 12(7), e1005775. https://doi.org/10.1371/journal.ppt.1005775.eCollection (2016).

9. Nywening, A. V., Rybak, J. M., Rogers, P. D. \& Fortwendel, J. R. Mechanisms of triazole resistance in Aspergillus fumigatus. Environ. Microbiol. 22, 4924-4952 (2020).

10. Van Ingen, J. et al. Azole, polyene and echinocandin, MIC distributions for wild-type, TR34/L98H and TR46/Y121F/T289A Aspergillus fumigatus isolates in the Netherlands. J. Antimicrob. Chemother. 70, 178-181 (2015).

11. Nathan, P. W. et al. First detection of TR34 L98H and TR46 Y121F T289A cyp51 mutations in Aspergillus fumigatus isolated in the United States. J. Clin. Microbiol. 54, 168-171 (2016).

12. Rößler, S. et al. Progressive dispersion of azole resistance in Aspergillus fumigatus: Fatal invasive aspergillosis in a patient with acute myeloid leukemia infected with an A. fumigatus strain with a cyp51A TR46 Y121FM1721 T289A allele. Antimicrob. Agents Chemother. 61(8), e00270. https://doi.org/10.1128/AAC.00270-17 (2017).

13. Rybak, J. M., Fortwendel, J. R. \& Rogers, P. D. Emerging threat of triazole-resistant Aspergillus fumigatus. J. Antimicrob. Chemother. 74, 835-842 (2019). 
14. Sharpe, A. R. et al. Triazole resistance surveillance in Aspergillus fumigatus. Med. Mycol. 56, S38-S92 (2018).

15. Inacio, J., Flores, O. \& Spencer-Martins, I. Efficient identification of clinical relevant Candida yeast species by use of an assay combining panfungal loop-mediated isothermal DNA amplification with hybridization to species-specific oligonucleotide probes. J. Clin. Microbiol. 46, 713-729 (2008).

16. Patrice, F. et al. Rubustness of a loop-nediated isothermal amplification reaction for diaganostic applification. FEMS Immunol. Med. Microbiol. 62, 41-48 (2011).

17. Shirato, K. Detecting amplification of loop-mediated isothermal amplification. Microbiol. Immunol. 63, 407-412 (2019).

18. Ling, S. Y. et al. Rapid detection of azole-resistant Aspergillus fumigatus in clinical and environmental isolates by use of a lab-ona-chip diagnostic system. J. Clin. Microbiol. 58, e00843 (2020).

19. Dudakova, A. et al. Molecular tools for the detection and deduction of azole antifungal drug resistance phenotypes in Aspergillus species. Clin. Microbiol. Rev. 30, 1065-1091 (2017).

20. Zhang, J. et al. A novel environmental azole resistance mutation in Aspergillus fumigatus and a possible role of sexual reproduction in its emergence. MBio 8(3), e00791. https://doi.org/10.1128/mBio.00791-17 (2017).

21. Hagiwara, D. Isolation of azole-resistant Aspergillus fumigatus from imported plant bulbs in Japan and the effect of fungicide treatment. J. Pestic. Sci. 45, 147-150 (2020).

22. Trabasso, P. et al. Isolation and drug susceptibility of Candida parapsilosis sensu lato and other species of $C$. parapsilosis complex from patients with blood stream infections and proposal of a novel LAMP identification method for the species. Mycopathology 179, 53-62 (2015).

23. de Souza, M. et al. Comparison of DNA microarray, loop-mediated isothermal amplification (LAMP) and real-time PCR with DNA sequencing for identification of Fusarium spp. obtained from patients with hematologic malignancies. Mycopathology 182, 625-632 (2017).

\title{
Acknowledgements
}

This study was supported by Japan Agency for Medical Research and Development (AMED) under Grant Number JP21jm0110015 and Japan International Cooperation Agency (JICA) through the collaborative research project Science and Technology Research Partnership for Sustainable Development (SATREPS), Japan under Grant Number 02-P-9427/2018.

\section{Author contributions}

P.T.: conception; methodological design; laboratory work; data collection; data analysis; writing; revising. T.M.: conception; methodological design; laboratory work; data collection; data analysis; writing, revising. T.A.:conception; methodological design; laboratory work; data collection; data analysis; writing, revising. D.H.: methodological design; laboratory work; data collection; writing. Y.M.: methodological design; writing; supervision. M.L.M.: critical review; team leadership; funding. A W.: critical review; team leadership; funding.

\section{Competing interests}

The authors declare no competing interests.

\section{Additional information}

Correspondence and requests for materials should be addressed to P.T.

Reprints and permissions information is available at www.nature.com/reprints.

Publisher's note Springer Nature remains neutral with regard to jurisdictional claims in published maps and institutional affiliations.

\begin{abstract}
(c) (i) Open Access This article is licensed under a Creative Commons Attribution 4.0 International cc) License, which permits use, sharing, adaptation, distribution and reproduction in any medium or format, as long as you give appropriate credit to the original author(s) and the source, provide a link to the Creative Commons licence, and indicate if changes were made. The images or other third party material in this article are included in the article's Creative Commons licence, unless indicated otherwise in a credit line to the material. If material is not included in the article's Creative Commons licence and your intended use is not permitted by statutory regulation or exceeds the permitted use, you will need to obtain permission directly from the copyright holder. To view a copy of this licence, visit http://creativecommons.org/licenses/by/4.0/.
\end{abstract}

(C) The Author(s) 2021 